Ciencia y Salud, Vol. II, No. 1, enero-abril, 2018 •ISSN: 2613-8816

\title{
FRECUENCIA Y MANEJO DE HEMATURIA Y PROTEINURIA EN PACIENTES ATENDIDOS EN LA EMERGENCIA DE PEDIATRÍA DEL HOSPITAL MATERNO INFANTIL SAN LORENZO DE LOS MINA, REPÚBLICA DOMINICANA, ABRIL DE 2016
}

\section{Frequency and management of hematuria and proteinuria in patients seen in the pediatric emergency of the San Lorenzo de Los Mina Maternal and Child Hospital, Dominican Republic, April 2016}

\author{
Willna de la Rosa Brito*, Manuel Guzmán**, Carlos Rodríguez***
}

Recibido: 4/7/2017 Aceptado: 30/8/2017

DOI: http://dx.doi.org/10.22206/cysa.2018.v2i1.pp57-62

\section{Resumen}

Se realizó un estudio descriptivo, prospectivo, de corte transversal, con el objetivo de establecer la frecuencia y el manejo de hematuria y proteinuria en pacientes atendidos en la emergencia de pediatría del Hospital Materno Infantil San Lorenzo de Los Mina, durante el mes de abril de 2016. Unos 54 pacientes cursaron con proteinuria o hematuria, lo que representa un $3.29 \%$ de los nińos y adolescentes atendidos durante ese período. Los pacientes masculinos (67\%) y los de edad entre 2-5 años (46.29\%) fueron los más afectados.

Los signos y síntomas agregados más frecuentes fueron vómitos, con un $42.59 \%$, y fiebre, con un $40.74 \%$. De los pacientes estudiados, el $40.74 \%$ cursó con amigdalitis como antecedente personal patológico; el $48.14 \%$ de sus familiares padece de hipertensión arterial. El método empleado para el diagnóstico fue la tira reactiva, dando como resultado un $77.77 \%$ con proteinuria, y un $14.81 \%$ para hematuria; el $7.40 \%$ de ellos presentó ambos hallazgos.

En conclusión, se debe dar seguimiento a los pacientes que diagnostiquemos con proteinuria o hematuria para evitar complicaciones renales crónicas.

Palabras clave: proteinuria; hematuria; niños; adolescentes; tira reactiva.

\footnotetext{
* Pediatra; Hospital Materno Infantil San Lorenzo de Los Mina, República Dominicana. Correo-e: willna25@hotmail.com

** Nefrólogo pediatra; Hospital Materno Infantil San Lorenzo de Los Mina, República Dominicana.
}

\section{Summary}

A prospective, cross-sectional study was carried out to determine the frequency and management of hematuria and proteinuria in patients attending the pediatric emergency at the San Lorenzo de Los Mina Maternal and Child Hospital during the month of April 2016. Some 54 patients had proteinuria or hematuria, representing $3.29 \%$ of the children and adolescents seen during that period. Males (67\%) patients and those aged 2-5 years (46.29\%) were the most affected. The most frequent signs and symptoms were vomiting, in $42.59 \%$, and fever, in $40.74 \%$. Of the patients studied, $40.74 \%$ had tonsillitis as a personal pathological antecedent, and $48.14 \%$ of their relatives had high blood pressure. The method used for the diagnosis was the test strip, resulting in $77.77 \%$ with proteinuria, and $14.81 \%$ for hematuria; $7.40 \%$ presented both findings.

In conclusion, patients who are diagnosed with proteinuria or hematuria should be followed to avoid chronic renal complications.

Keywords: proteinuria; hematuria; children; adolescents; tira reactive.

\footnotetext{
*** Asesor de investigación.

Correo-e: hguzman3.mhgz@gmail.com
} 


\section{Introducción}

La hematuria ${ }^{1,2}$ y la proteinuria ${ }^{3}$ son hallazgos frecuentes en el análisis de orina en la infancia. Ocurren típicamente en tres situaciones: en pacientes con síntomas específicos, por ejemplo, hematuria macroscópica o Síndrome nefrótico; en pacientes sin síntomas específicos (generalmente fiebre), y en niños asintomáticos. La mayoría de los niños entran en los dos últimos grupos, el hallazgo es temporario y no está asociado con enfermedad renal a largo plazo.

Si el hallazgo es persistente o los pacientes tienen características clínicas específicas, entonces las anomalías renales son más frecuentes y se requiere una investigación apropiada ${ }^{4,5}$.

La pérdida de proteínas en la orina es uno de los principales indicadores de enfermedad renal en niños y adolescentes, que según la definición de la OMS es la etapa que transcurre entre los $10 \mathrm{y}$ los 19 ańos. La proteinuria persistente puede ser un indicador de enfermedad renal evidente y también el primer indicador de un daño renal silente. Es igualmente un determinante de enfermedad renal crónica (ERC) y un factor de riesgo de progresión de esta y, al igual que en adultos, es un factor de riesgo cardiovascular. Pero, el enfoque de la proteinuria es diferente en adultos y niños porque en estos puede estar condicionada por trastornos benignos y ser transitoria, además de tener otras etiologías ${ }^{6}$.

La tira reactiva es útil para la detección de posible proteinuria. Es sensible y específica en un $99 \%$ para la albuminuria, pero no para otro tipo de proteínas (no detectan proteínas de bajo peso molecular). El método más sencillo para la cuantificación de la proteinuria y el más útil para el pediatra de cabecera es el cociente proteína/ creatinina $(\mathrm{Pr} / \mathrm{Cr})(\mathrm{mg} / \mathrm{mg})$ en orina, patológico si $>0,2$ ( $>0,5$ en niños de 6-24 meses). Es importante que sea la primera orina de la mañana, así excluiremos también proteinuria ortostática. Para el control de niños diabéticos es mejor la medición de la microalbuminuria, ya que es más sensible. Los valores superiores a 30 de albúmina/ creatinina $(\mathrm{mg} / \mathrm{g})$ en la primera orina de la mañana son considerados anormales ${ }^{7}$.

La presencia de sangre en la orina de un niño, sea macro o microscópica, es un motivo de consulta frecuente que puede ocasionar gran ansiedad en la familia. Es la anomalía urinaria más común, ya que la gran mayoría de las enfermedades que afectan al aparato urinario cursarán en algún momento de su evolución con hematuria. Puede presentarse de forma muy variable, desde la presencia alarmante de hematuria macroscópica asociada a diversos síntomas hasta una micro hematuria asintomática descubierta como un hallazgo casual.

\section{Material y métodos}

El estudio se realizó en la emergencia del Hospital Materno Infantil San Lorenzo de Los Mina, en la ciudad de Santo Domingo, República Dominicana.

El universo estuvo constituido por 3,451 pacientes atendidos en la emergencia de pediatría del hospital, en abril de 2016. De estos, 1,641 pacientes tenían edad de escolares y adolescentes, y solo 54 casos $(3.29 \%)$ presentaron hematuria y/o proteinuria, determinada mediante tira reactiva de orina al momento de la consulta. Estos constituyen nuestro objeto de estudio y en ellos evaluamos las siguientes variables: edad, sexo, proteinuria, hematuria, signos y síntomas, antecedentes personales y familiares, y método diagnóstico.

Los datos obtenidos en el estudio fueron presentados en frecuencia simple y las variables son susceptibles de comparación.

\section{Resultados}

El 77.77\% de los casos (42/54) presentó proteinuria; un $14.81 \%$ (8/54), hematuria; y 4 casos $(7.40 \%)$, proteinuria y hematuria a la vez (ver gráfico 1 ). 
Gráfico 1: Frecuencia de hematuria y proteinuria en pacientes atendidos en la emergencia de pediatría del Hospital Materno Infantil San Lorenzo de Los Mina, en abril de 2016.

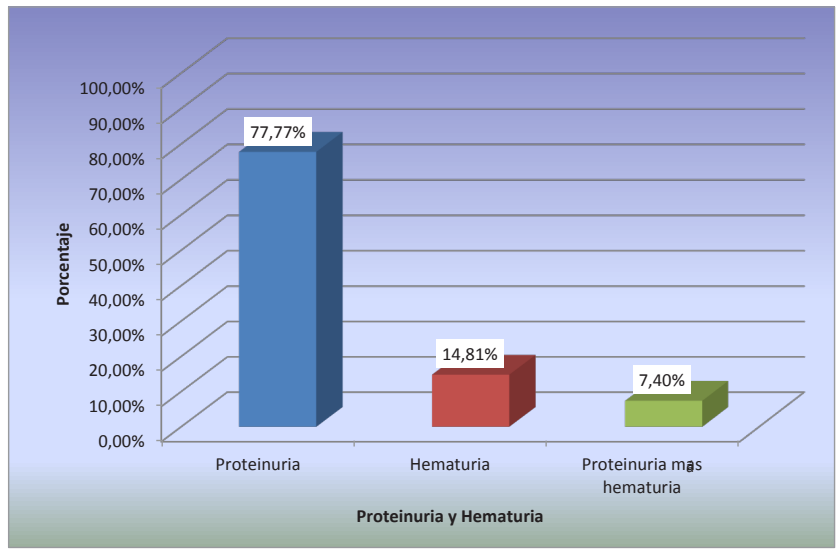

Los grupos de edades de 2-5 años (41.29\%) y de 6-10 ańos (40.74\%) fueron los más afectados, y el sexo masculino predominó, con un $67 \%$ (ver gráficos 2 y 3 ).

Gráfico 2: Edad de aparición más frecuente de hematuria y proteinuria en pacientes atendidos en la emergencia de pediatría del Hospital Materno Infantil San Lorenzo de Los Mina, en abril de 2016.

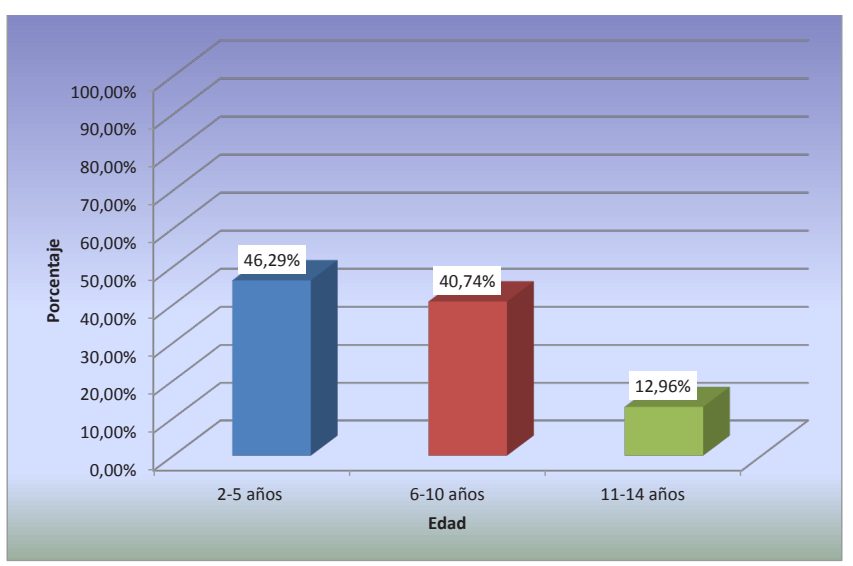

Gráfico 3: Sexo más frecuente de los pacientes diagnosticados con la hematuria y proteinuria atendidos en la emergencia de pediatría del Hospital Materno Infantil San Lorenzo de Los Mina, en abril de 2016.

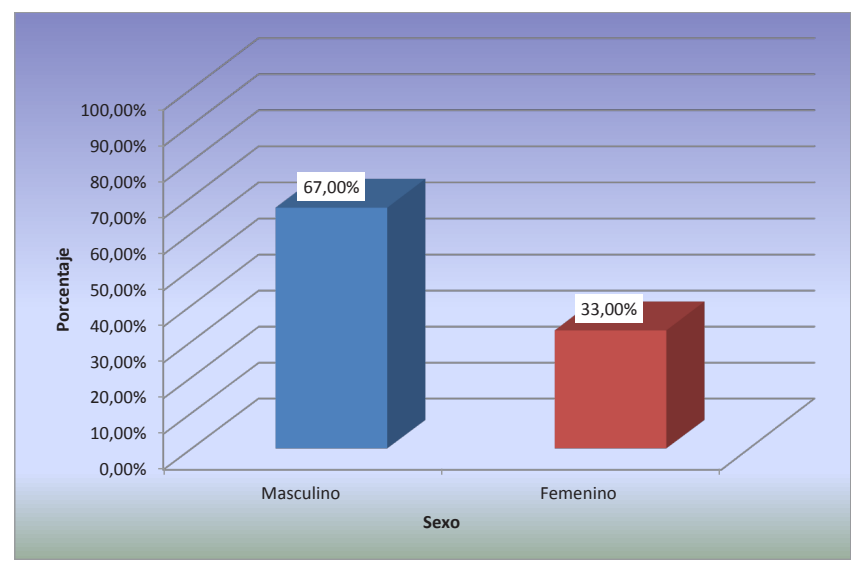

Los signos y síntomas de los pacientes al establecer el diagnóstico de hematuria y proteinuria en la emergencia de pediatría del Hospital Materno Infantil San Lorenzo de Los Mina, en abril de 2016, se expresan en el gráfico 4.

Gráfico 4: Signos y síntomas agregados más frecuentes de los pacientes diagnosticados con hematuria y proteinuria en la emergencia de pediatría del Hospital Materno Infantil San Lorenzo de Los Mina, en abril de 2016.

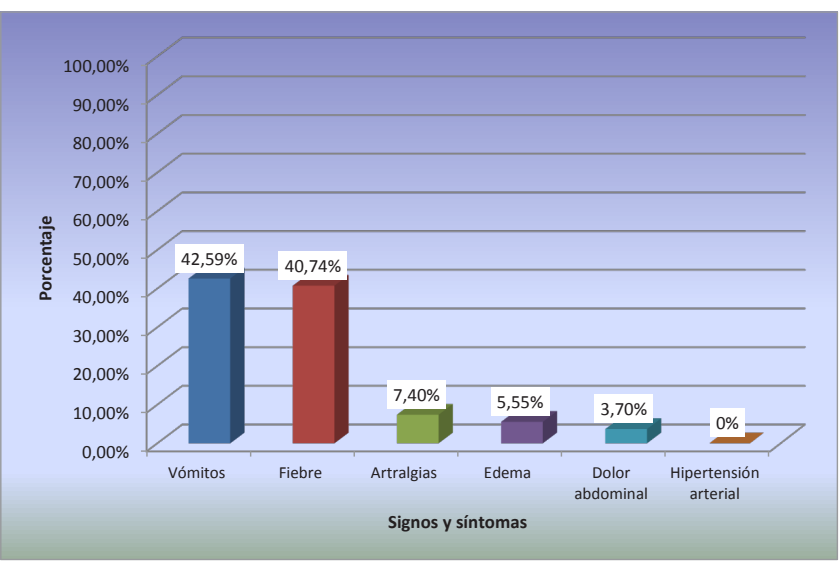


Los antecedentes personales patológicos que con mayor frecuencia padecen los pacientes diagnosticados con hematuria y proteinuria, atendidos en la emergencia de pediatría, correspondieron a infección de vías respiratorias e hipertensión arterial, que fue el principal hallazgo entre los familiares (ver gráficos 5 y 6 ).

Gráfico 5: Antecedentes personales patológicos que con mayor frecuencia padecen los pacientes diagnosticados con hematuria y proteinuria, atendidos en la emergencia de pediatría del Hospital Materno Infantil San Lorenzo de Los Mina, en abril de 2016

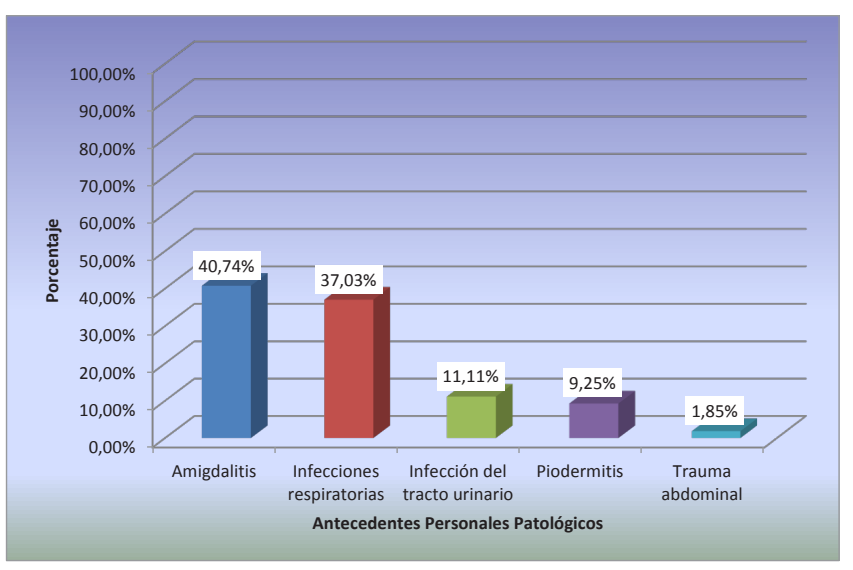

Gráfico 6: Antecedentes heredo familiares de los pacientes diagnosticados con hematuria y proteinuria, atendidos en la emergencia de pediatría del Hospital Materno Infantil San Lorenzo de Los Mina, en abril de 2016

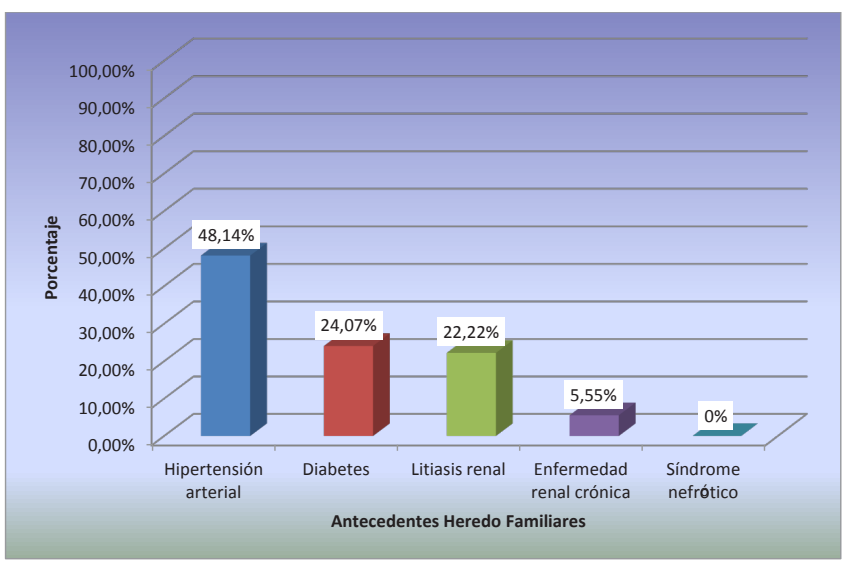

\section{Discusión}

La hematuria y la proteinuria son signos de enfermedad de las vías urinarias que se encuentran con relativa frecuencia en la orina de los niños ${ }^{8,9}$. Las causas son muy variadas: puede tratarse de trastornos transitorios, de patología renal de evolución benigna, o bien que exista una enfermedad renal más severa subyacente.

La categorización, según la forma clínica de presentación, puede ser de gran ayuda para el pediatra decidir cuáles exámenes complementarios son los más indicados en cada situación para investigar la etiología ${ }^{7}$.

Se estima que la prevalencia de este trastorno entre la población sana se sitúa entre el 0,5 y el $2 \%$, y en una reciente revisión de programas de cribaje urinario llevada a cabo en países asiáticos, con muestras de hasta 5 millones de escolares, se ha constatado la presencia de hematuria aislada hasta en el 0,94\% de los niños ${ }^{3}$.

En nuestro estudio se identificaron 54 pacientes, para un $3.29 \%$ de los atendidos en la emergencia de pediatría, quienes cumplieron con los criterios de inclusión y exclusión. Se identificó un $77.77 \%$ de pacientes con proteinuria; un $14.81 \%$, con hematuria, y ambos hallazgos en un $7.40 \%$ de los pacientes.

En el estudio realizado en Barcelona por Tauler Girona $^{7}$, en 2013, se demuestra que la asociación hematuria-proteinuria tiene una prevalencia inferior al $0,7 \%$. Mientras que la incidencia de hematuria macroscópica es de un $0,13 \%$.

Varios estudios en niños en edad escolar han mostrado que el promedio de prevalencia de la hematuria microscópica detectada en una muestra simple de orina es de 3 a $4 \%$. Dentro del $1 \%$ de niños con 2 o más análisis de orina positivo para hematuria, solo un tercio tiene hematuria persistente, definida como positiva luego de la repetición de la prueba 6 
meses más tarde. La combinación de hematuria y proteinuria es comúnmente baja, con un promedio de prevalencia $<0,7 \%$ en nińos en edad escolar, en una muestra simple de orina.

Una circunstancia particular es el hallazgo de una hematuria microscópica asintomática en un niño aparentemente sano, casi siempre en el curso de una visita rutinaria a consultas o emergencias. Estudios obligatorios de tamizaje urinario realizados en Japón a más de 500,000 niños han establecido que su prevalencia es de $0.54 \%$ en niños de 6-11 años, $y$ de $0.94 \%$ en niños de $11-13$ años de edad ${ }^{3}$.

En nuestro estudio analizamos que, del total de pacientes, el $46.29 \%$ correspondía a niños entre 2 y 5 años de edad, y un $40.74 \%$ a escolares de 6-10 ańos, dándonos a demostrar que el mayor porcentaje de pacientes con proteinuria o hematuria están en la edad preescolar y escolar.

Con relación al sexo más frecuente establecido en nuestro estudio, de los pacientes que cursaron con proteinuria o hematuria, se encontró un $67 \%$ para el sexo masculino, equivalente a 36 pacientes de la muestra total; correlacionándose este con estudios antes realizados que muestran que el mayor porcentaje de los afectados corresponde a los varones.

En un estudio realizado sobre hematuria en niños en un centro de referencia nacional en Venezuela, se demostró que durante el período de estudio fueron evaluados 362 pacientes con hematuria, lo cual representa el $8,4 \%$ de las consultas nefrológicas pediátricas, y el $1,1 \%$ de las consultas o admisiones realizadas en su centro hospitalario, durante el mismo lapso. La edad promedio de los pacientes fue $7,7 \pm 6,1$ años, con unrangode 0 a 17 años. Encuanto al sexo, 203 correspondieron al masculino (56\%) y 159 al femenino $(44 \%)^{10}$.

La hematuria tiene una multiplicidad de causas. Habitualmente, es un síntoma transitorio de poca significación, pero en ocasiones puede ser el único síntoma de una enfermedad grave y que requiere una actuación urgente. Se precisa conocer las características de la hematuria macroscópica (coloración, relación con la micción, duración), así como las circunstancias acompañantes (antecedentes de traumatismo abdominal o lumbar, Síndrome miccional, ejercicio previo, antecedente de infección respiratoria o cutánea). Las infecciones dérmicas o respiratorias padecidas 1-3 semanas antes del comienzo de la hematuria sugieren una glomerulonefritis aguda postestreptocócica.

Los hallazgos clínicos como oliguria, anuria, edemas e hipertensión arterial sugieren hematuria de origen glomerular; si además se detecta proteinuria es probable que nos encontremos ante una glomerulonefritis. La palidez cutánea, expresión de edema y/o anemia, puede asociarse a hipertensión arterial o Síndrome hemolítico urémico. La disuria, micción imperiosa, polaquiuria y dolor en flanco sugieren que la hematuria tiene su origen en uretra, vejiga o uréter.

Se puede presentar signos y síntomas acompañando la hematuria, sea macroscópica o microscópica, así como también asociados a la proteinuria; en nuestro estudio, investigamos pacientes que nos llegaban a la emergencia por sintomatologías diversas e identificamos que el $40.74 \%$ acudió por fiebre y como hallazgo fortuito encontramos proteinuria o hematuria; un $42.59 \%$ acudió con historia de vómitos, $5.55 \%$ de ellos presentaron edema, ninguno de los pacientes estudiados presentó hipertensión arterial y solo hubo un paciente por trauma abdominal, para un $1.85 \%$.

Es importante interrogar por antecedentes de consanguinidad, familiares cercanos con historia de proteinuria o hematuria, litiasis, sordera, hipertensión arterial, insuficiencia renal crónica, diálisis o trasplante; especialmente se debe indagar en la historia de afectación renal con malformaciones asociadas (como el Síndrome de uña-rótula), ya que muchas de las causas de proteinuria y hematuria pueden ser de origen hereditario y por razones 
genéticas; por esto, resulta importante hacer un buen interrogatorio a los padres sobre los antecedentes heredo familiares del niño.

En nuestro estudio pudimos observar que como antecedente familiar 26 pacientes $(48.14 \%)$ cursaban con antecedentes de hipertensión arterial de diferentes causas, un $22.22 \%$ con litiasis renal, 3 de los estudiados (5.55\%) con enfermedad renal crónica, demostrándonos que 15 de nuestros pacientes tienen familiares con patologías renales.

La tira reactiva (Dipstick) es el test más utilizado para el screening de hematuria. Es sencillo y barato; detecta sangre basándose en la actividad peroxidasa de la hemoglobina. Es útil en la hematuria microscópica. Tiene una sensibilidad de un 100\% y una especificidad aproximada de un 99\%. La tira detecta de 5-10 hematíes intactos/ $\mathrm{mm} 3$, que corresponde a $2-5$ hematíes/campo en sedimento de orina de una muestra centrifugada. Además, es útil para la detección de posible proteinuria. Es sensible y específica en un $99 \%$ para la albuminuria, pero no para otro tipo de proteínas (no detectan proteínas de bajo peso molecular?

En varios estudios realizados, la detección de proteinuria se hace a través de tiras reactivas, o se cuantifica con el índice proteinuria/creatininuria $(\mathrm{P} / \mathrm{C})$ o albuminuria/creatininuria $(\mathrm{A} / \mathrm{C})$ en una micción. Estos valores se correlacionan bien con la medida de proteínas en orina de 24 horas, que correctamente realizada constituye el patrón oro para su detección ${ }^{6}$.

Nuestra observación corrobora con los datos emitidos en diversos estudios donde se evidencia que el $98.14 \%$ de los pacientes estudiados presenta proteinuria o hematuria detectada por la tira reactiva, enviándose luego a realizar examen de orina y proteinuria de 24 horas, según la sintomatología presentada.

La presente revisión revela la importancia de detectar a tiempo la hematuria y la proteinuria por parte del pediatra general, permitiendo un manejo adecuado en el consultorio o en la emergencia. Se debe considerar la importancia de un adecuado seguimiento clínico y de laboratorio de primera línea, y tener presente los signos y síntomas de alarma para una derivación precoz al nefrólogo pediátrico, en caso de ser necesario.

\section{Bibliografía}

1. Lunn A, Forbes TA. Haematuria and proteinuria in childhood. Pediatr Child Health. 2012; 22(8): 315-321.

2. Mena E. Hematuria en niños. Archivos dominicanos de pediatría. 1995; 31(2): 59-68.

3. Ariceta G. Clinical practice: Proteinuria. Eur J Pediatric. 2011; 170(1): 15-20.

4. Hui-Kim Y, Perry YW. Hematuria and Proteinuria. In: Geary D, Schaefer F. Comprehensive Pediatric Nephrology. Philadelphia: Elsevier; 2008. 1040 p.

5. Collantes C, Izquierdo E. Proteinuria. Revista Nefrología Pediátrica, protocolo diagnóstico. 2014; 1: 69-79.

6. Verocay MC, Rebori A, Velasco M. Significado de la proteinuria en el niño y adolescente. Archivos médicos internacionales. 2012; 34(1): 12-16.

7. Girona T. Hematuria, proteinuria: actitud diagnóstica. Pediatría integral. 2013; 17(6): 412-421.

8. Piña J, Saieh C. Hematuria en pediatría. Revista médico clínica Condes. 2009; 20(6): 904-910.

9. Escalante C, Zeledon F, Ulate G. Proteinuria, fisiología y fisiopatología aplicada. Acta médica costarricense. 2007; 49(2): 83-89.

10. Cruz M. Diagnóstico diferencial de las hema-

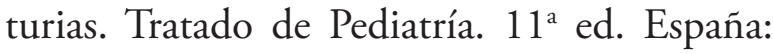
Editora Médica Panamericana. 2600 p. 